\title{
A rigorous inexact Newton method with applications to Boundary Value Problems
}

\author{
Eduardo Ramost \\ Instituto de Ciências Matemáticas e de Computação, USP, São Carlos, SP \\ Victor Hugo Nolascop \\ Instituto de Ciências Matemáticas e de Computação, USP, São Carlos, SP \\ Marcio Gameird $\beta^{3}$ \\ Instituto de Ciências Matemáticas e de Computação, USP, São Carlos, SP
}

\begin{abstract}
We introduce a semi-local theorem for the feasibility and convergence of the inexact Newton method $u_{k+1}=u_{k}-D \mathcal{F}\left(u_{k}\right)^{-1} \mathcal{F}\left(u_{k}\right)+r_{k}$, where $r_{k}$ represents the error in each step. Unlike the previous results of this type in the literature, we prove the feasibility of the inexact Newton method under the minor hypothesis that the error $r_{k}$ is bounded by a small constant to be computed, and moreover we prove results concerning the convergence of the sequence $u_{k}$ to the solution under this hypothesis. We present an application of the method to rigorously compute zeros for two-point boundary value problems.
\end{abstract}

Keywords. Differential equations, Inexact Newton Method, Newton-Kantorovich Theorem, Rigorous Numerics

\section{Introduction}

The celebrated Newton-Kantorovich theorem [6] provides (semi-local) sufficient conditions for the convergence of the Newton method $u_{k+1}=u_{k}-D \mathcal{F}\left(u_{k}\right)^{-1} \mathcal{F}\left(u_{k}\right)$ to a zero of $\mathcal{F}$. On one hand this is a very powerful and useful result and can be used to compute rigorous bounds to guarantee the existence of solutions, on the other hand the exact Newton method is usually impracticable for the purpose of rigorous computations since in general we cannot expect to be able to perform the computations of $u_{k}-D \mathcal{F}\left(u_{k}\right)^{-1} \mathcal{F}\left(u_{k}\right)$ without numerical errors. These numerical errors can be incorporated in the computations by means of error bounds, but in general cannot be avoided, specially in the infinite dimensional setting. One option to circumvent this problem is to consider the more general inexact Newton method $u_{k+1}=u_{k}-D \mathcal{F}\left(u_{k}\right)^{-1} \mathcal{F}\left(u_{k}\right)+r_{k}$ in which $r_{k}$ represents the numerical error during each step of the computation.

In the recent literature, such as [1 $1: 2,5,8,9]$, semi-local conditions for the inexact Newton method to be feasible and convergent have been given in the form $\left\|r_{k}\right\| \leq \eta\left\|\mathcal{F}\left(u_{k}\right)\right\|$ under conditions on the bounding term $\eta$. In that case, since the sequence $u_{k}$ is know to converge fast to the zeros of $\mathcal{F}$, it follows that $\mathcal{F}\left(u_{k}\right)$ converges fast to 0 and thus the same must hold for error term $r_{k}$. This fast decay rate for $r_{k}$, however, is a very strong condition which is hard to obtain in actual applications.

With this in mind, we present in this paper a condition for the feasibility under the minor condition that the error terms $r_{k}$ are bounded by a small constant $d$ to be computed, and we

\footnotetext{
${ }^{1}$ eduardoramos@usp.br

2 victor.nolasco@usp.br

3 gameiro@icmc.usp.br
} 
prove convergence of the sequence $u_{k+1}=u_{k}-D \mathcal{F}\left(u_{k}\right)^{-1} \mathcal{F}\left(u_{k}\right)+r_{k}$ under the condition $r_{k} \rightarrow 0$ as $k \rightarrow \infty$, with no additional requirements regarding the rate of convergence of $r_{k}$. Moreover, together with the Newton-Kantorovich theorem and the bijectivity modulus [7, we present a method to rigorously compute solutions to boundary value problems.

\section{Inexact Newton method}

In this section we present a version of the Newton-Kantorovich theorem aimed to the Inexact Newton method.

Definition 2.1 (Newton operator). Let $X$ and $Y$ be Banach spaces, $U \subset X$ open, and $\mathcal{F}: U \subset$ $X \rightarrow Y$ differentiable in $U$. We define the Newton operator $T_{\mathcal{F}}: X \rightarrow X$ by $T_{\mathcal{F}}(u)=u-$ $D \mathcal{F}(u)^{-1} \mathcal{F}(u)$ if $u \in U$ and $D \mathcal{F}(u)$ is invertible, and $T_{\mathcal{F}}(u)=0$ otherwise.

Definition 2.2 (Feasibility for Newton method). Let $X$ and $Y$ be Banach spaces, $U \subset X$ open, $A \subset U, \mathcal{F}: U \subset X \rightarrow Y$ differentiable in $U$, and let $\left\{u_{k}\right\}_{k \in \mathbb{N}}$ be a sequence in $X$. We say that $\left\{u_{k}\right\}_{k \in \mathbb{N}}$ is feasible for the Newton method for $\mathcal{F}$ in $A$, if $u_{k} \in A$ and $D \mathcal{F}\left(u_{k}\right)$ is invertible for all $k \in \mathbb{N}$.

Thus, from the above definitions, if $\left\{u_{k}\right\}_{k \in \mathbb{N}}$ is feasible for the Newton method in $A \subset U$, and $\left\{u_{k}\right\}_{k \in \mathbb{N}}$ satisfies the recurrence relation $u_{k+1}=T_{\mathcal{F}}\left(u_{k}\right)$ for all $k \in \mathbb{N}$, then $u_{k+1}=u_{k}-$ $D \mathcal{F}\left(u_{k}\right)^{-1} \mathcal{F}\left(u_{k}\right)$, for all $k \in \mathbb{N}$, which corresponds to the classical Newton method. Based on the Newton-Kantorovich theorem we propose the following theorem regarding the feasibility and convergence of the Inexact Newton method.

Theorem 2.1. Let $X$ and $Y$ be Banach spaces, $U \subset X$ an open set, $\mathcal{F}: U \subset X \rightarrow Y$ a differentiable function in $U, u_{0} \in U, R>0$ satisfying $\bar{B}\left(u_{0}, R\right) \subset U$, let $\left\{r_{k}\right\}_{k \in \mathbb{N}}$ be a sequence in $X$, and let $\eta \geq 0, K>0$ and $d>0$. Suppose that

(a) $D \mathcal{F}\left(u_{0}\right)$ is invertible with $D \mathcal{F}\left(u_{0}\right)^{-1} \in \mathcal{L}(Y, X)$.

(b) $\left\|D \mathcal{F}\left(u_{0}\right)^{-1} \mathcal{F}\left(u_{0}\right)\right\| \leq \eta$ and $\left\|D \mathcal{F}\left(u_{0}\right)^{-1}(D \mathcal{F}(u)-D \mathcal{F}(v))\right\| \leq K\|u-v\|$ for all $u, v \in \bar{B}\left(u_{0}, R\right)$.

(c) $\left\|r_{k}\right\| \leq d$ for all $k \in \mathbb{N}$.

(d) $g_{d}(t)=\eta_{d}-(1+K d) t+\frac{3 K}{2} t^{2}$ has a smallest real zero $t_{d}^{*} \leq R$ and moreover $d \leq \frac{1}{K}$, where $\eta_{d}=\eta+d$

Then $0<t_{d}^{*} \leq \frac{1}{K}\left(1-\frac{1}{\sqrt{3}}\right)$ and moreover

(i) The function $\mathcal{F}$ has a unique zero $u^{*} \in \bar{B}\left(u_{0}, t_{d}^{*}\right)$.

(ii) The sequence $\left\{u_{k}\right\}_{k \in \mathbb{N}}$ defined by $u_{k+1}=T_{\mathcal{F}}\left(u_{k}\right)+r_{k}$ for all $k \in \mathbb{N}$ is feasible for the Newton method for $\mathcal{F}$ in $\bar{B}\left(u_{0}, t_{d}^{*}\right)$.

(iii) $\left\|u^{*}-u_{k+1}\right\| \leq \frac{\sqrt{3}}{2} K\left\|u^{*}-u_{k}\right\|^{2}+\left\|r_{k}\right\|$ for all $k \in \mathbb{N}$.

(iv) If $\lim _{k \rightarrow \infty}\left\|r_{k}\right\|=0$, then $\lim _{k \rightarrow \infty} u_{k}=u^{*}$. 
Proof. It is easy to see that

$$
0<t_{d}^{*} \leq \frac{1}{K}\left(1-\frac{1}{\sqrt{3}}\right)
$$

Proof of $(i)$ : Given any $z \in \bar{B}\left(u_{0}, t_{d}^{*}\right)$ such that $D \mathcal{F}(z)$ is invertible, we define $L_{z}: U \rightarrow X$ by $L_{z}(u)=u-D \mathcal{F}(z)^{-1} \mathcal{F}(u)$ for all $u \in U$. We shall prove in the following that $L_{u_{0}}$ is a contraction from $\bar{B}\left(u_{0}, t_{d}^{*}\right)$ to $\bar{B}\left(u_{0}, t_{d}^{*}\right)$. Thus, due to the Banach fixed point theorem (see 4. Theorem 2.1, p. $24]$ ), follows that $L_{u_{0}}$ has a unique fixed point, which in turn proves item $(i)$ since it is clear that every zero of $\mathcal{F}$ in $\bar{B}\left(u_{0}, t_{d}^{*}\right)$ is a fixed point of $L_{u_{0}}$ and vice-versa.

The proof that $L_{u_{0}}$ is a contraction on $\bar{B}\left(u_{0}, t_{d}^{*}\right)$ will follow from the verification of the three items bellow,

(I) $\left\|L_{u_{0}}(u)-L_{u_{0}}(v)\right\| \leq c\|u-v\|$ for all $u, v \in \bar{B}\left(u_{0}, t_{d}^{*}\right)$ for some $c<1$.

(II) $D \mathcal{F}(z)$ is invertible for all $z \in \bar{B}\left(u_{0}, t_{d}^{*}\right)$ with $\left\|D \mathcal{F}(z)^{-1} D \mathcal{F}\left(u_{0}\right)\right\| \leq \frac{1}{1-K t_{d}^{*}}$.

(III) $L_{z}\left(\bar{B}\left(u_{0}, t_{d}^{*}\right)\right) \subset \bar{B}\left(u_{0}, t_{d}^{*}-d\right)$ for all $z \in \bar{B}\left(u_{0}, t_{d}^{*}\right)$.

Proof of $(i i)$ : Since $L_{z}(z)-u_{0}=z-D \mathcal{F}(z)^{-1} \mathcal{F}(z)-u_{0}$ it follows that if $r \in X$ with $\|r\| \leq d$ then due to item $(I I I)$ above we have $\left\|z-D \mathcal{F}(z)^{-1} \mathcal{F}(z)+r-u_{0}\right\| \leq\left\|u_{0}-L_{z}(z)\right\|+d \leq t_{d}^{*}$. Combining this with the results obtained in the proof of $(i)$ we can conclude that, given $z \in \bar{B}\left(u_{0}, t_{d}^{*}\right)$, it follows that $D \mathcal{F}(z)$ is invertible and $z-D \mathcal{F}(z)^{-1} \mathcal{F}(z)+r \in \bar{B}\left(u_{0}, t_{d}^{*}\right)$ for all $r \in X$ with $\|r\| \leq d$. Thus, using induction, we can conclude that the sequence $\left\{u_{k}\right\}_{k \in \mathbb{N}}$ defined by $u_{k+1}=T_{\mathcal{F}}\left(u_{k}\right)+r_{k}$ for all $k \in \mathbb{N}$ is such that $D \mathcal{F}\left(u_{k}\right)$ is invertible and $u_{k} \in \bar{B}\left(u_{0}, t_{d}^{*}\right)$ for all $k \in \mathbb{N}$, which proves $(i i)$.

Proof of $\left(\right.$ iii): Let $L_{u_{m}}$ be defined by $L_{u_{m}}(u)=u-D \mathcal{F}\left(u_{m}\right)^{-1} D \mathcal{F}(u)$ as in the proof of item $(i)$. Then it follows that $u^{*}=u^{*}-D \mathcal{F}\left(u_{m}\right)^{-1} \mathcal{F}\left(u^{*}\right)$, and we can show that we have

$$
\left\|u^{*}-u_{m+1}\right\|=\left\|L_{u_{m}}\left(u^{*}\right)-L_{u_{m}}\left(u_{m}\right)-r_{m}\right\| \leq \frac{K\left\|u^{*}-u_{m}\right\|^{2}}{2\left(1-K t_{d}^{*}\right)}+\left\|r_{m}\right\| .
$$

Now, we can show that

$$
K t_{d}^{*} \leq 1-\frac{1}{\sqrt{3}} \Rightarrow \frac{1}{2\left(1-K t_{d}^{*}\right)} \leq \frac{\sqrt{3}}{2}
$$

Thus we conclude from $\sqrt{1}$ and $\sqrt{2}$ that $\left\|u^{*}-u_{m+1}\right\| \leq \frac{\sqrt{3}}{2} K\left\|u^{*}-u_{m}\right\|^{2}+\left\|r_{m}\right\|$ which proves item $($ iii).

Proof of $(i v)$ : Given $m \in \mathbb{N}$, since we proved $K t_{d}^{*} \leq 1-\frac{1}{\sqrt{3}}$ and since from $(i i)$ we have that $u_{m} \in \bar{B}\left(u_{0}, t_{d}^{*}\right)$ it follows that $\frac{\sqrt{3}}{2} K\left\|u^{*}-u_{m}\right\| \leq \frac{\sqrt{3}}{2} K t_{d}^{*} \leq \frac{\sqrt{3}-1}{2}$. Thus, from item (iii) it follows that, for all $m \in \mathbb{N},\left\|u^{*}-u_{m+1}\right\| \leq \frac{\sqrt{3}}{2} K\left\|u^{*}-u_{m}\right\|^{2}+\left\|r_{m}\right\| \leq\left(\frac{\sqrt{3}-1}{2}\right)\left\|u^{*}-u_{m}\right\|+\left\|r_{m}\right\|$. Now, given $\epsilon>0$, by definition of liminf there exists $N \in \mathbb{N}$ such that $k \geq N$ implies $\left\|r_{k}\right\| \leq \epsilon$. Thus, considering the sequence $\left\{h_{k}\right\}_{k \geq N}$ defined by recurrence via

$$
h_{N}=\left\|u^{*}-u_{N}\right\| \text { and } h_{k+1}=h_{k}\left(\frac{\sqrt{3}-1}{2}\right)+\epsilon \text { for all } k \geq N
$$

it follows from inequality (2) that $\left\|u^{*}-u_{k}\right\| \leq h_{k}$ for all $k \geq N$. On the other hand, one can verify via induction that the recurrence above implies in the formula $h_{N+k}=h_{N}\left(\frac{\sqrt{3}-1}{2}\right)^{k}+$ $\sum_{m=0}^{k-1} \epsilon\left(\frac{\sqrt{3}-1}{2}\right)^{m}$ for all $k \in \mathbb{N}$ and thus $\lim _{k \rightarrow \infty} h_{k}=\sum_{m=0}^{\infty} \epsilon\left(\frac{\sqrt{3}-1}{2}\right)^{m}=\frac{\epsilon}{1-\left(\frac{\sqrt{3}-1}{2}\right)}=\left(1+\frac{1}{\sqrt{3}}\right) \epsilon$.

Therefore, since $\left\|u^{*}-u_{k}\right\| \leq h_{k}$ for all $k \geq N$, it follows that $\lim \inf _{k \rightarrow \infty}\left\|u^{*}-u_{k}\right\| \leq\left(1+\frac{1}{\sqrt{3}}\right) \epsilon$ and since $\epsilon>0$ was arbitrary chosen, letting $\epsilon \rightarrow 0^{+}$in the above inequality, the result follows. 


\section{Bijectivity Modulus and reformulation of the Main Result}

To apply the Theorem 2.1 to rigorously verify the existence of a true zero for a partial differential equation near a numerical zero, we will use the bijectivity modulus defined below.

Definition 3.1. Let $X$ and $Y$ be Banach spaces. For $F \in \mathcal{L}(X, Y)$ we define the bijectivity modulus $\lambda(F)$ of $F$ by $\lambda(F)=\left\|F^{-1}\right\|^{-1}$ if $F$ is invertible, and $\lambda(F)=0$ otherwise.

Theorem 3.1. Let $X$ and $Y$ be Banach spaces, $U \subset X$ an open set, $\mathcal{F}: U \subset X \rightarrow Y$ a differentiable function in $U, u_{0} \in U, R>0$ satisfying $\bar{B}\left(u_{0}, R\right) \subset U$, let $\left\{r_{k}\right\}_{k \in \mathbb{N}}$ be a sequence in $X$, and let $\eta \geq 0, \nu \geq 0, K>0$ and $d>0$. Suppose that

(a) $\left\|\mathcal{F}\left(u_{0}\right)\right\| \leq \eta, \lambda\left(D \mathcal{F}\left(u_{0}\right)\right) \geq \nu$ and $\|D \mathcal{F}(u)-D \mathcal{F}(v)\| \leq K\|u-v\|$ for all $u, v \in \bar{B}\left(u_{0}, R\right)$.

(b) $\left\|r_{k}\right\| \leq d$ for all $k \in \mathbb{N}$.

(c) $g_{d}(t)=\eta_{d}-\nu_{d} t+\frac{3 K}{2} t^{2}$ has a a smallest real zero $t_{d}^{*} \leq R$ and moreover $d \leq \frac{\nu}{K}$, where $\eta_{d}=\eta+\nu d$ and $\nu_{d}=\nu+K d$.

Then $\nu>0,0<t_{d}^{*} \leq \frac{\nu}{K}\left(1-\frac{1}{\sqrt{3}}\right)$ and moreover

(i) The function $\mathcal{F}$ has a unique zero $u^{*} \in \bar{B}\left(u_{0}, t_{d}^{*}\right)$.

(ii) The sequence $\left\{u_{k}\right\}_{k \in \mathbb{N}}$ defined by $u_{k+1}=T_{\mathcal{F}}\left(u_{k}\right)+r_{k}$ for all $k \in \mathbb{N}$ is feasible for the Newton method for $\mathcal{F}$ in $\bar{B}\left(u_{0}, t_{d}^{*}\right)$.

(iii) $\left\|u^{*}-u_{k+1}\right\| \leq \frac{\sqrt{3}}{2} K \nu^{-1}\left\|u^{*}-u_{k}\right\|^{2}+\left\|r_{k}\right\|$ for all $k \in \mathbb{N}$.

(iv) If $\lim _{k \rightarrow \infty}\left\|r_{k}\right\|=0$, then $\lim _{k \rightarrow \infty} u_{k}=u^{*}$.

$(v)\left\|v-T_{\mathcal{F}}\left(u_{k}\right)\right\| \leq \sqrt{3} \nu^{-1}\left\|\mathcal{F}\left(u_{k}\right)-D \mathcal{F}\left(u_{k}\right)\left(v-u_{k}\right)\right\|$ for all $v \in U$ and $k \in \mathbb{N}$.

Proof. Proof of (i) to (iv): Notice that from hypothesis $0<d \leq \frac{\nu}{K}$ and therefore $\nu>0$. Thus it follows that $\lambda\left(D \mathcal{F}\left(u_{0}\right)\right) \geq \nu>0$, which by definition implies that $D \mathcal{F}\left(u_{0}\right)$ is invertible with $\left\|D \mathcal{F}\left(u_{0}\right)^{-1}\right\|=\lambda\left(D \mathcal{F}\left(u_{0}\right)\right)^{-1} \leq \nu^{-1}$. Therefore, letting $\eta^{*}=\frac{\eta}{\nu}$ and $K^{*}=\frac{K}{\nu}$ it follows that $\left\|D \mathcal{F}\left(u_{0}\right)^{-1} \mathcal{F}\left(u_{0}\right)\right\| \leq\left\|D \mathcal{F}\left(u_{0}\right)^{-1}\right\|\left\|\mathcal{F}\left(u_{0}\right)\right\| \leq \eta^{*}$ and $\left\|D \mathcal{F}\left(u_{0}\right)^{-1}(D \mathcal{F}(u)-D \mathcal{F}(v))\right\| \leq$ $\left\|D \mathcal{F}\left(u_{0}\right)^{-1}\right\|\|(D \mathcal{F}(u)-D \mathcal{F}(v))\| \leq K^{*}\|u-v\|$ for all $u, v \in A$. Thus, the hypothesis $(a)$ and $(b)$ of Theorem 2.1 are satisfied. Therefore, letting $\eta_{d}^{*}=\eta^{*}+\nu^{*} d$ and $\eta_{d}^{*}=\eta^{*}+K^{*} d$ from item $(i i)$ it follows that the polynomial $g_{d}^{*}(t)=\eta_{d}^{*}-\nu_{d}^{*} t+\frac{3 K^{*}}{2} t^{2}=\nu^{-1} g_{d}(t)$ has a smallest zero $t_{d}^{*} \leq R$, and thus from Theorem 2.1 items $(i)$ to $(i v)$ follows.

Proof of $(v)$ : Notice that $\left|\lambda\left(D \mathcal{F}\left(u_{k}\right)\right)-\lambda\left(D \mathcal{F}\left(u_{0}\right)\right)\right| \leq\left\|D \mathcal{F}\left(u_{k}\right)-D \mathcal{F}\left(u_{0}\right)\right\| \leq K\left\|u_{k}-u_{0}\right\| \leq$ $K t_{d}^{*} \leq \nu\left(1-\frac{1}{\sqrt{3}}\right) \Rightarrow \lambda\left(D \mathcal{F}\left(u_{k}\right)\right) \geq \lambda\left(D \mathcal{F}\left(u_{0}\right)\right)-\nu\left(1-\frac{1}{\sqrt{3}}\right) \geq \nu-\nu\left(1-\frac{1}{\sqrt{3}}\right)=\frac{\nu}{\sqrt{3}}>0$, and thus $\left\|v-T_{\mathcal{F}}\left(u_{k}\right)\right\|=\left\|D \mathcal{F}\left(u_{k}\right)^{-1}\left(\mathcal{F}\left(u_{k}\right)-D \mathcal{F}\left(u_{k}\right)\left(u_{k}-v\right)\right)\right\| \leq \frac{\sqrt{3}}{\nu}\left\|\mathcal{F}\left(u_{k}\right)-D \mathcal{F}\left(u_{k}\right)\left(u_{k}-v\right)\right\|$, proving the theorem.

Remark 3.1. Supposing the hypothesis of Theorem 3.1 is true and letting $\left\{u_{k}\right\}_{k \leq n}$ be such that $u_{k+1}=T_{\mathcal{F}}\left(u_{k}\right)+r_{k}$ and $\left\|r_{k}\right\| \leq d$ for all $k<n$, we can let $r_{k}=0$ for all $k \geq n$ and use the conclusions of Theorem 3.1. In special, $(v)$ can be used to estimate $\left\|v-T_{\mathcal{F}}\left(u_{n}\right)\right\|$ for any $v \in U$, and thus it can be used to estimate $\left\|u_{k+1}-T_{\mathcal{F}}\left(u_{k}\right)\right\|$ where $u_{n+1}$ is the candidate for new term in the Inexact Newton Method. 


\section{Applications}

We apply Theorem 3.1 to use the Inexact Newton Method for two point boundary value problems of the form

$$
u^{\prime \prime}=f(x, u), \quad u^{\prime}(0)=u^{\prime}(1)
$$

where $f: \mathbb{R}^{2} \rightarrow \mathbb{R}$. Letting $I=(0,1)$ and $I^{\prime}=[0,1]$, it is known that $H^{2}(I)$ can be regarded as $C^{1}\left(I^{\prime}\right)$ functions (see 3 . Theorem 8.2]), we let $H_{N}^{2}(I)=\left\{u \in H^{2}(I) \mid u^{\prime}(0)=u^{\prime}(1)\right\}$. Then zeros $u \in H^{2}(I)$ of the two point boundary value problem in (3) are the zeros of the operator $\mathcal{F}: H_{N}^{2}(I) \rightarrow L^{2}(I)$ defined by

$$
\mathcal{F}(u)=u^{\prime \prime}-f(x, u) .
$$

Letting $\omega(k):=\sqrt{1+((k-1) \pi)^{2}+((k-1) \pi)^{4}}$ for $k \in \mathbb{N}$, then $\pi_{\cos }: L^{2}(I) \rightarrow \ell^{2}(\mathbb{N})$ and $h_{\cos }:$ $H_{N}^{2}(I) \rightarrow \ell^{2}(\mathbb{N})$ defined by $\pi_{\cos }(u)=\left(\widehat{u}_{\cos }(1), \widehat{u}_{\cos }(2), \cdots\right)$ and $h_{\cos }(u)=\left(\omega(1) \widehat{u}_{\cos }(1), \omega(2) \widehat{u}_{\cos }(2), \cdots\right)$ are isometric isomorphisms, where $\left\{\widehat{u}_{\cos }(k)\right\}_{k \in \mathbb{N}}$ is the sequence of coefficients of $u \in L^{2}(I)$ in the cosine basis (see $\left[3\right.$ p. 145]). Thus, we define $\pi_{\cos , m}: L^{2}(I) \rightarrow \mathbb{R}^{m}$ by $\pi_{\cos , m}(u)=$ $\left(\widehat{u}_{\cos }(1), \cdots, \widehat{u}_{\cos }(m)\right)$ and $h_{\cos , m}^{-1}: \mathbb{R}^{m} \rightarrow H_{N}^{2}(I)$ the restrictions to $\mathbb{R}^{m} \subset \ell^{2}(\mathbb{N})$, where we identify $\mathbb{R}^{m}$ with a subset of $\ell^{2}(\mathbb{N})$ through the isometric embedding $\pi_{\mathbb{R}^{m}}(a)=\left(a_{1}, \cdots, a_{m}, 0, \cdots\right) \in \ell^{2}(\mathbb{N})$, for $a=\left(a_{1}, \cdots, a_{m}\right) \in \mathbb{R}^{m}$. Given an operator $\mathcal{G}: H_{N}^{2}(I) \rightarrow L^{2}(I)$, it is reasonable to consider the finite dimensional operator $\mathcal{G}_{\cos , m}: \mathbb{R}^{m} \rightarrow \mathbb{R}^{m}$ defined by $\mathcal{G}_{\text {cos }, m}=\pi_{\cos , m} \circ \mathcal{G} \circ h_{\mathrm{cos}, m}^{-1}$ as a natural finite dimensional approximation for $\mathcal{G}$.

Given $m>0$, and $f: \mathbb{R}^{2} \rightarrow \mathbb{R}$ a $C^{2}$ function, and an initial point $u_{0}=h_{\text {cos }, m}^{-1}\left(b_{0}\right) \in H_{N}^{2}(I)$, where $b_{0} \in \mathbb{R}^{m}$, to compute $\eta \geq 0, \nu \geq 0, K>0$ in Theorem 3.1, we follow the reference [7]. The sequence $\left\{u_{k}\right\}_{k \in \mathbb{N}}$ for the Inexact Newton Method for $\mathcal{F}$ in $H_{N}^{2}(I)$ can be chosen computing nonrigorously the sequence $\left\{b_{k}\right\}_{k \in \mathbb{N}}$ for the Newton Method $b_{k+1}=T_{\mathcal{F}_{\text {cos }, m}}\left(b_{k}\right), b_{0}=h_{\cos }\left(u_{0}\right) \in \mathbb{R}^{m}$ for the finite dimensional function $\mathcal{F}_{\cos , m}: \mathbb{R}^{m} \rightarrow \mathbb{R}^{m}$ and then letting $u_{k}=h_{\cos , m}^{-1}\left(b_{k}\right)$ for all $k \in \mathbb{N}$. Since all $u_{k}$ will be elementary functions, it follows that rigorous enclosures for the errors $\left\|r_{k}\right\|=\left\|u_{k+1}-T_{\mathcal{F}}\left(u_{k}\right)\right\|$ can be computed using the Simpson's rule with rigorous error bounds using the error formula given by item $(v)$ of Theorem 3.1 , that is

$$
\begin{aligned}
\left\|u_{k+1}-T_{\mathcal{F}}\left(u_{k}\right)\right\| & \leq \sqrt{3} \nu^{-1}\left\|\mathcal{F}\left(u_{k}\right)-D \mathcal{F}\left(u_{k}\right)\left(u_{k+1}-u_{k}\right)\right\| \\
& =\sqrt{3} \nu^{-1}\left\|-u_{k+1}^{\prime \prime}+2 u_{k}^{\prime \prime}-f\left(x, u_{k}\right)-f_{u}\left(x, u_{k}\right)\left(u_{k+1}-u_{k}\right)\right\|
\end{aligned}
$$

Example. Consider the boundary value problem

$$
u^{\prime \prime}=\sin (u(x))-\cos (2 \pi x), \quad u^{\prime}(0)=u^{\prime}(1) .
$$

Then $\mathcal{F}: H_{N}^{2}(I) \rightarrow L^{2}(I)$ is the operator given by $\mathcal{F}(u)(x)=u^{\prime \prime}(x)-(\sin (u(x))-\cos (2 \pi x))$ for all $u \in H_{N}^{2}(I)$ and $x \in I$. It was shown in $[7$ that, for $m=5$,

$$
b_{0}=\left(3.14159265328020,0,0.734842599198155,0,2.19859211843525 \times 10^{-11}\right)
$$

is an approximate zero of $\mathcal{F}_{\cos , 5}$ in $\bar{B}(0,1)_{\mathbb{R}^{5}}$, with $\left\|\mathcal{F}_{\cos , 5}\left(b_{0}\right)\right\|=8.185852625229473 \times 10^{-9}$, and thus

$$
u_{0}=h_{\cos }^{-1}\left(b_{0}\right)=b_{0}(1)+\sum_{k=2}^{m} b_{0}(k) \frac{\sqrt{2} \cos ((k-1) \pi x)}{w(k)}
$$

is an approximate zero for $\mathcal{F}$ in $\bar{B}(0,1)_{H_{N}^{2}(I)}$. A plot of the numerical solution $u_{0}$ is shown in Figure 1. Following [7] we computed $\eta=0.57075361344299 \times 10^{-6}, \nu=0.29866890113073$, and $K=1$ as valid constants for Theorem 3.1 .

Finally, letting $d=10^{-5}$ we obtain a zero $t_{d}^{*}$ for $g_{d}(t)=\eta_{d}-\nu_{d} t+\frac{3 K}{2} t^{2}$ satisfying $t_{d}^{*}=$ $1.19113048496 \times 10^{-5} \leq 1$. Thus, the conditions of Theorem 3.1 are satisfied and we can apply the Inexact Newton Method with garanteed feasibility. 


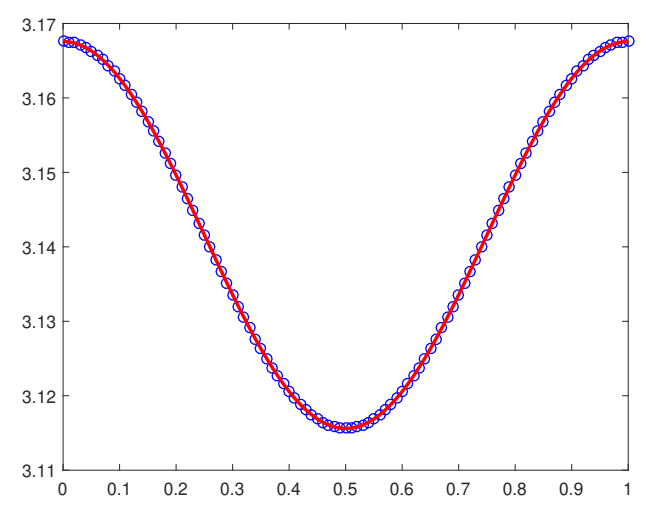

Figure 1: Approximate solutions $u_{0}$ (solid red curve) and $u_{4}$ (blue circles).

The iterates $u_{k}$ for the Inexact Newton Method are computed using $u_{k}=h_{\text {cos }}^{-1}\left(b_{k}\right)$, where $b_{k}$ are the iterates in the Newton Method for $\mathcal{F}_{\mathrm{cos}, 5}$ starting at $b_{0}$. Using equation (5) the errors $r_{k}=u_{k+1}-T_{\mathcal{F}}\left(u_{k}\right)$, are proven to satisfy

$$
\left\|r_{k}\right\| \leq d, \quad \text { for } 0 \leq k \leq 4
$$

The norms of these itearations are shown on Table 1. From item $(i)$ of Theorem 3.1 we conclude that there exists an unique zero $u^{*}$ of $\mathcal{F}$ in $\bar{B}\left(u_{0}, t_{d}^{*}\right)_{H_{N}^{2}(I)}$. Moreover, we obtain by Inexact Newton Method $u_{4}$ such that $\left\|u^{*}-u_{4}\right\| \leq 2.9989923490307 \times 10^{-6}<t_{d}^{*}$, that is, $u_{4}$ is closer to the true solution $u^{*}$ than $u_{0}$ and $u_{4} \in \bar{B}\left(u_{0}, t_{d}^{*}\right)_{H_{N}^{2}(I)}$. Thus, using our method, it was possible to obtain a better approximation for a zero of $\mathcal{F}$ compared to the one obtained in 7 .

Table 1: Iterations of the initial solution $u_{0}$ with the Inexact Newton method. The first column shows the norm of $\mathcal{F}$, the second column presents the norm of the error terms, and the third column shows the distances from the computed solutions to the true solution $u^{*}$.

\begin{tabular}{cccc}
\hline$k$ & $\left\|\mathcal{F}_{\cos , 5}\left(b_{k}\right)\right\|$ & $\left\|r_{k}\right\|$ & $\left\|u^{*}-u_{k}\right\|$ \\
\hline 0 & $8.185852625229473 \times 10^{-9}$ & - & $t_{d}^{*}=1.19113048496 \times 10^{-5}$ \\
\hline 1 & $1.184138216374711 \times 10^{-16}$ & $3.00046899391681 \times 10^{-6}$ & $3.0008803891992 \times 10^{-6}$ \\
\hline 2 & $1.171296009783492 \times 10^{-16}$ & $2.99896627000569 \times 10^{-6}$ & $2.9989923818771 \times 10^{-6}$ \\
\hline 3 & $1.150312379208693 \times 10^{-16}$ & $2.99896627000465 \times 10^{-6}$ & $2.9989923490297 \times 10^{-6}$ \\
\hline 4 & $1.140972793438999 \times 10^{-16}$ & $2.99896627000624 \times 10^{-6}$ & $2.9989923490307 \times 10^{-6}$ \\
\hline \hline
\end{tabular}

\section{Conclusion}

In this paper, we propose a new theorem for the feasibility and convergence of the inexact Newton method, with explicit convergence rate formulas similar to that of the Newton-Kantorovich theorem. We use this theorem, together with our definition of bijectivity modulus, to rigorosuly verify the existence of zeros of a differential operator $\mathcal{F}$ using the inexact Newton method. 
We illustrate the method with an example, where we computed a solution to a boundary value problem. It was shown that given an initial solution, the method can compute a more accurate solution in a few steps.

\section{References}

[1] Argyros, I.K., Saïd Hilout, Ángel A. Magreñán. Robust semi-local convergence analysis for inexact Newton method, Applied Mathematics and Computation, 227:741-754, 2014. DOI: 10.1016/j.amc.2013.11.076.

[2] Argyros, I.K., George, S., Senapati, K. Extending the applicability of the inexact Newton-HSS method for solving large systems of nonlinear equations, Numerical Algorithms, 83:333-353, 2020. DOI: $10.1007 / \mathrm{s} 11075-019-00684-\mathrm{z}$.

[3] Brezis, H. Functional Analysis, Sobolev Spaces and Partial Differential Equations. SpringerVerlag New York, New York, 2011. DOI: 10.1007/978-0-387-70914-7.

[4] Chow, S. N., Hale, J. K. Methods of bifurcation theory. Springer Science and Business Media, Grundlehren der mathematischen Wissenschaften, volume 251, number 1, 1982. DOI: $10.1007 / 978-1-4613-8159-4$.

[5] Ferreira, O. P., Svaiter, B. F. A robust Kantorovich's theorem on the inexact Newton method with relative residual error tolerance. Journal of Complexity, 28(3):346-363, 2012. DOI: 10.1007/10.1016/j.jco.2012.02.002.

[6] Kantorovich, L. V., Akilov, G. P. Functional analysis second edition. Pergamon Press, 1982. DOI: 10.1016/C2013-0-03044-7.

[7] Ramos, E., Gameiro, M., Nolasco, V. Rigorous Enclosures of Solutions of Neumann Boundary Value Problems, arXiv preprint arXiv:2005.02755v2, 2020. DOI: to appear.

[8] Shen, W., Li, C. Kantorovich-type convergence criterion for inexact Newton methods, Applied Numerical Mathematics, 59(7):1599-1611, 2009. DOI: 10.1016/j.apnum.2008.11.002.

[9] Wu, M. A new semi-local convergence theorem for the inexact Newton methods. Applied mathematics and computation, 200(1):80-86, 2008. DOI: 10.1016/j.amc.2007.10.057. 